\title{
Design of a Minimized Complementary Illusion Cloak with Arbitrary Position
}

\author{
Yan Shi, Lin Zhang, Wei Tang, Long Li, and Chang-Hong Liang \\ School of Electronic Engineering, Xidian University, Xian, Shaanxi 710071, China \\ Correspondence should be addressed to Yan Shi; shiyan@mail.xidian.edu.cn
}

Received 15 May 2015; Revised 6 July 2015; Accepted 7 July 2015

Academic Editor: Shah N. Burokur

Copyright (C) 2015 Yan Shi et al. This is an open access article distributed under the Creative Commons Attribution License, which permits unrestricted use, distribution, and reproduction in any medium, provided the original work is properly cited.

\begin{abstract}
This paper has developed a complementary illusion cloak to hide the objects with arbitrary shapes and sizes. In the proposed cloak, a complementary cloak is first introduced by using the trapezoid coordinate transformation. Then an illusion device in terms of another trapezoid coordinate transformation is designed to cloak the object. With two coordinate transformations, the complementary illusion cloak device can be arbitrarily placed to hide the target object, and the sizes of the antiobject used to cloak the object are obviously compressed. Numerical results including target objects with different shapes are given to verify correctness and effectiveness of the proposed cloak.
\end{abstract}

\section{Introduction}

In recent years, transformation optics-based invisibility cloaks have attracted considerable interests. Making use of form-invariant coordinate transformation of Maxwell's equations, Pendry et al. [1] and Leonhardt [2] independently proposed the invisibility cloak and the concept of transformation media for electromagnetic (EM) waves. Lately, some key issues related to the coordinate transformation theory including ray tracing [3], numerical simulation [4], singularity treatment [5], and embedded coordinate transformations [6] have been addressed, and various invisibility cloaks have been proposed, for instance, elastic cloaking devices [7], conformally invisible cloaks [8], three-dimensional cloaks [9], and nonmagnetic optical cloak [10]. Following this success, the coordinate transformation theory has resulted in the development of interesting devices including superscatterer [11], concentrators [12], and transparent metamaterial slabs [13]. Besides, practical implementations of the invisibility cloaks have been discussed [14, 15]. However, one of disadvantages of the conventional invisibility cloaks is that the object hidden inside the cloaked domain is "blind," since no exterior electromagnetic waves can reach into the cloaked space. The concept of the complementary media $[16,17]$ provides us with a new idea to construct an invisibility cloak which can hide the objects external to the cloak itself. The complementary media, most notably in the "perfect lens" [18], can optically "cancel" a certain volume of space at a certain frequency. By locating a complementary "image" of the object embedded in a negative index shell, which is called the "antiobject," an object as well as the surrounding space is optically canceled. Further, the correct optical path in the canceled space is restored by a dielectric core material, as shown in Figure 1. We call this kind of cloaks "open cloaks" and then the conventional cloaks are defined as "closed cloaks." Based on the complementary media, various open cloaks with different shapes [19], illusion cloaks [20, 21], and anticloaks with arbitrary shapes [22] have been developed. However, because of the implementation of the optical transformation along one direction, the required antiobject in these open cloaks can only be compressed along the corresponding direction, while the dimensions of the antiobject along other directions keep unchanged. Hence, the miniaturization of the open cloak cannot be achieved. More importantly, the relative position between the transformation region of the open cloak and the target object is fixed when the optical transformation along one direction is employed. In this scenario, the position of the cloak device cannot be arbitrarily changed.

In this paper, we propose a trapezoid complementary illusion cloak to hide the objects with arbitrary shapes and 


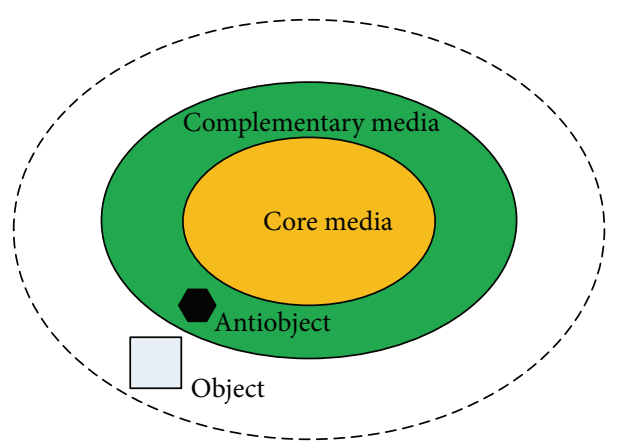

FIGURE 1: The external cloak based on the complementary media.

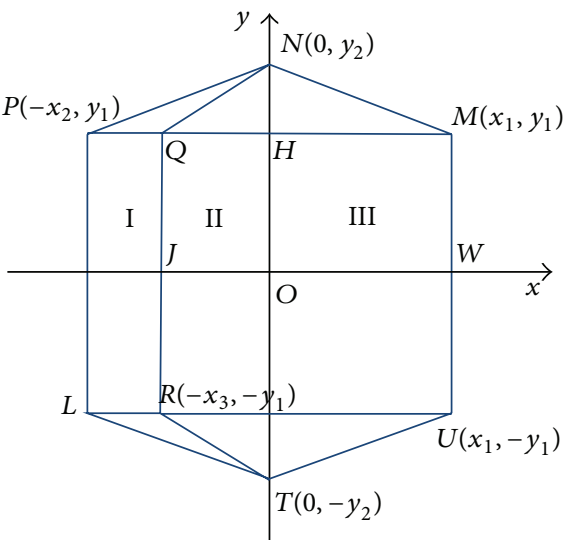

(a)

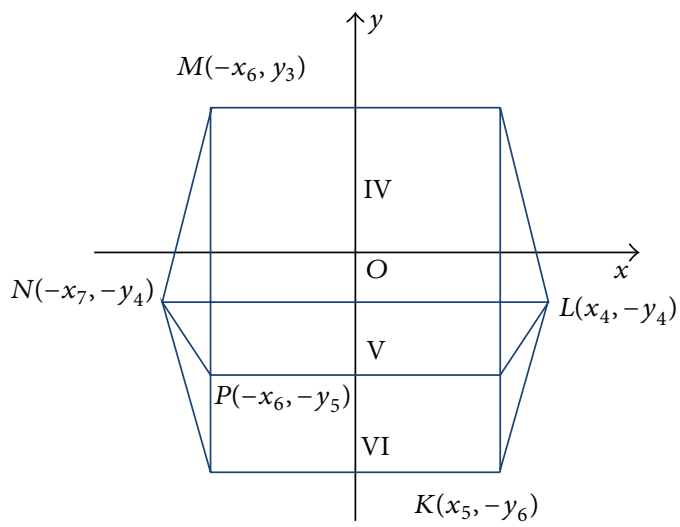

(b)

FIGURE 2: The transformation device: (a) the transformation along $x$-axis and (b) the transformation along $y$-axis.

sizes. In the proposed illusion cloak, a coordinate transformation along one direction is first conducted to design a trapezoid complementary cloak. With the complementary cloak, a coordinate transformation along another direction is further implemented to generate a new illusion cloak device used to cloak the target objects. Considering the coordinate transformations along two different directions, the sizes of the required antiobjects are greatly reduced for a target object with arbitrary size and shape. Moreover for a given target object, we can arbitrarily design the location of the illusion complementary cloak to hide the target object by using two coordinate transformations.

\section{Design of a Complementary Illusion Cloak}

According to the coordinate transformation theory $[1,2]$, when a space is transformed into another space of different shape and size, the permittivity tensor $\varepsilon^{\prime}$ and permeability tensor $\mu^{\prime}$ in the transformed space or physical space $X^{\prime}$ can be expressed according to the permittivity tensor $\varepsilon$ and permeability tensor $\mu$ in the original space $X$ as $\varepsilon^{\prime}=$ $\Lambda \varepsilon \Lambda^{T} / \operatorname{det}(\Lambda)$ and $\mu^{\prime}=\Lambda \mu \Lambda^{T} / \operatorname{det}(\Lambda)$, where $\Lambda$ is the Jacobian transformation tensor with components $\Lambda_{p q}=\partial p / \partial q(p=$ $\left.x^{\prime}, y^{\prime}, z^{\prime} ; q=x, y, z\right)$ and $\operatorname{det}(\Lambda)$ is determinant of $\Lambda$.

Consider a two-dimensional complementary cloak, as shown in Figure 2(a), where region I acts as the restoring medium and regions II and III are used as a pair of complementary media. In the complementary cloak, the transformations from region III to region II and from the whole region including regions I, II, and III to region I are implemented. In order to easily carry out the corresponding transformations, we divide each region into three smaller regions, for example, the upper region, the middle region, and the lower region. Specifically, three subregions in each of three regions can be, respectively, marked as $\mathrm{I}^{\text {upper }}, \mathrm{I}^{\text {middle }}$, $\mathrm{I}^{\text {lower }}, \mathrm{II}^{\text {upper }}, \mathrm{II}^{\text {middle }}, \mathrm{II}^{\text {lower }}, \mathrm{III}^{\text {upper }}, \mathrm{III}^{\text {middle }}$, and $\mathrm{III}^{\text {lower }}$. Hence, the coordinate transformations between III and II are divided into three transformations including two triangle region transformations and one rectangle region transformation. The coordinate transformations between the whole region and region I can be similarly handled. It can be seen from Figure 2(a) that transformations from region III to region II and from the whole region to region I are the linear transformations along $x$-axis.

The linear transformation from region III to region II can be uniformly expressed as

$$
\begin{aligned}
& x^{(2)}=A x^{(3)}+B y^{(3)}+C, \\
& y^{(2)}=D x^{(3)}+E y^{(3)}+F .
\end{aligned}
$$


Specifically, as to the transformation from region III $^{\text {middle }}$ to region $\mathrm{II}^{\text {middle }}$, the points $M, W$, and $H$ are mapped to points $Q, J$, and $H$, respectively, and thus we get $A=-x_{3} / x_{1}$, $E=1$, and $B=C=D=F=0$. With the similar mapping, we can get the transformations from region III ${ }^{\text {upper }}$ to region II $^{\text {upper }}$ and from region III $^{\text {lower }}$ to region II $^{\text {lower }}$ with $A=-x_{3} / x_{1}, E=1$, and other parameters of zero. If region III is air, the permittivity tensor and the permeability tensor in region II are diagonal matrices and can be expressed as follows:

$$
\varepsilon^{\prime(\mathrm{II})}=\mu^{\prime(\mathrm{II})}=\left[\begin{array}{ccc}
A & 0 & 0 \\
0 & \frac{1}{A} & 0 \\
0 & 0 & \frac{1}{A}
\end{array}\right]=\left[\begin{array}{ccc}
-\frac{x_{3}}{x_{1}} & 0 & 0 \\
0 & -\frac{x_{1}}{x_{3}} & 0 \\
0 & 0 & -\frac{x_{1}}{x_{3}}
\end{array}\right] .
$$

With the similar transformation process between the whole region and region I, the resultant material parameters of regions $\mathrm{I}^{\text {upper }}, \mathrm{I}^{\text {middle }}$, and $\mathrm{I}^{\text {lower }}$ can be

$$
\varepsilon^{\prime(\mathrm{I}) \mathrm{reg}}=\mu^{\prime(\mathrm{I}) \mathrm{reg}}=\left(\begin{array}{ccc}
\frac{A^{2}+B^{\mathrm{reg} 2}}{A} & \frac{B^{\mathrm{reg}}}{A} & 0 \\
\frac{B^{\mathrm{reg}}}{A} & \frac{1}{A} & 0 \\
0 & 0 & \frac{1}{A}
\end{array}\right),
$$

where

$$
\begin{aligned}
A & =\frac{x_{2}-x_{3}}{x_{1}+x_{2}}, \\
B^{\text {reg }} & \\
& = \begin{cases}\frac{x_{2}-x_{3}}{x_{1}+x_{2}} \cdot \frac{x_{1}}{y_{2}-y_{1}}+\frac{x_{3}}{y_{2}-y_{1}} & \text { reg }=\text { upper }, \\
0 & \text { reg }=\text { middle, } \\
-\frac{x_{2}-x_{3}}{x_{1}+x_{2}} \cdot \frac{x_{1}}{y_{2}-y_{1}}-\frac{x_{3}}{y_{2}-y_{1}} & \text { reg }=\text { lower. }\end{cases}
\end{aligned}
$$

From (2) and (3), we can clearly see that the cloak is made of a homogenous material, and the material parameters in entire complementary region and rectangular core region are just diagonally homogeneous, whereas only triangular core regions have anisotropic tensor properties. When there has an object with the permittivity of $\varepsilon$ and permeability of $\mu$ in region III, it can be cloaked by placing an antiobject in region II, of which material parameters can be determined by $\varepsilon^{\prime}=\Lambda \varepsilon \Lambda^{T} / \operatorname{det}(\Lambda)$ and $\mu^{\prime}=\Lambda \mu \Lambda^{T} / \operatorname{det}(\Lambda)$. In addition, the transformation from the whole region to region I makes region I optically equivalent to the whole region. In this scenario, the electromagnetic response produced by an object in region I is the same as that caused by another object with different size and material parameters in the whole region, which is called the illusion.

It can be seen from the above procedure that in the design of the complementary cloak the coordinate transformation

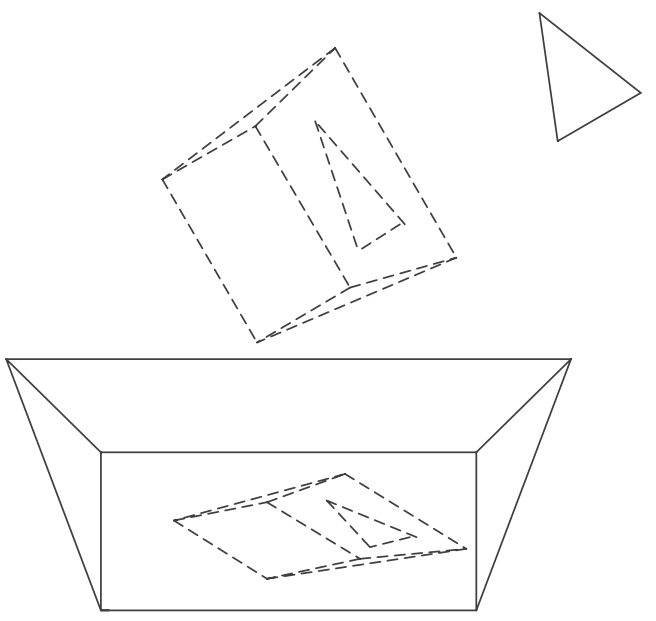

FIGURE 3: Schematic model to cloak a target object.

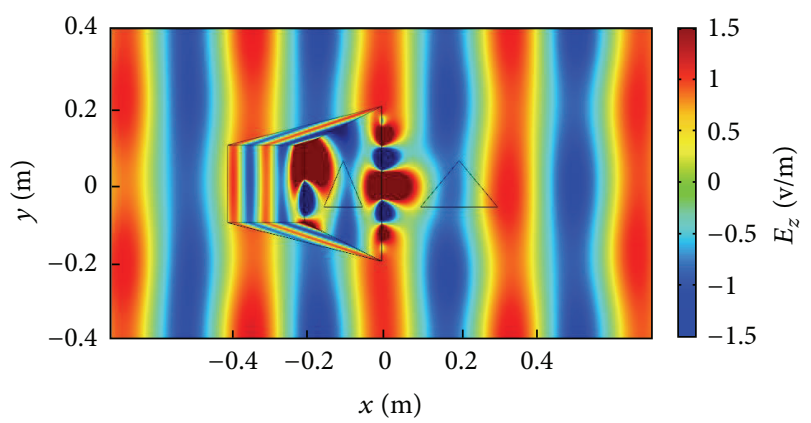

FIGURE 4: A trapezoid complementary cloak used to cloak a triangle object outside the cloak.

is only implemented along one direction, for example, $x$ direction, and therefore the resultant whole transformation region and the required antiobject can be only compressed along $x$ direction, which hinders the miniaturization of the whole device. Moreover, the use of only one coordinate transformation imposes restrictions on the relative position between the cloak and the target object. In order to solve these problems, two coordinate transformations along two different directions are proposed. Specifically, following above steps a coordinate transformation along a direction, for instance, $y$ direction, is first implemented to achieve a trapezoid complementary cloak, in which region VI acts as the restoring medium, and regions $\mathrm{V}$ and IV are chosen as a pair of complementary media, as shown in Figure 2(b). And then a cloak device used to hide the object is illusively generated to become a new complementary cloak based on the coordinate transformation along another direction. In order to generate the illusion device used to hide the object, the illusion object related to the illusion device is required to locate in region VI. The shapes, locations, and material parameters of the illusion object can be exactly determined according to the coordinate transformation from the whole region including regions IV, V, and VI to region VI, which is similar to the coordinate transformation given by (1). Hence, an object in region III is cloaked by placing an 


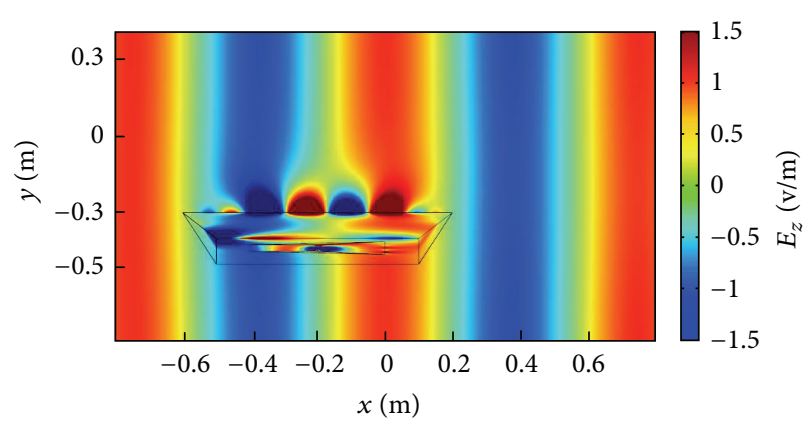

(a)

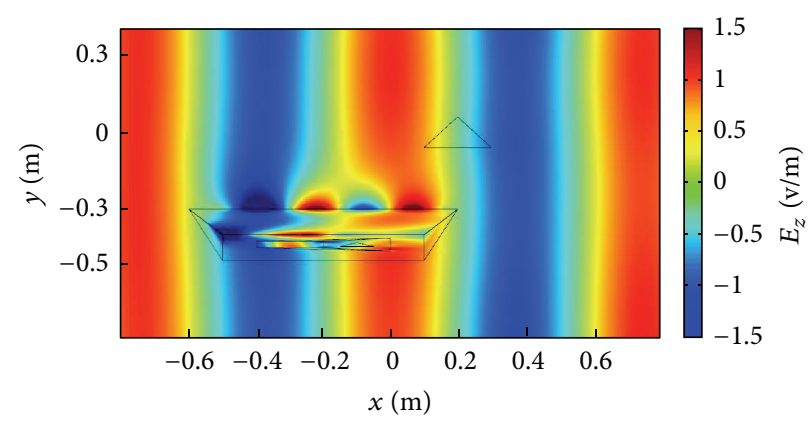

(b)

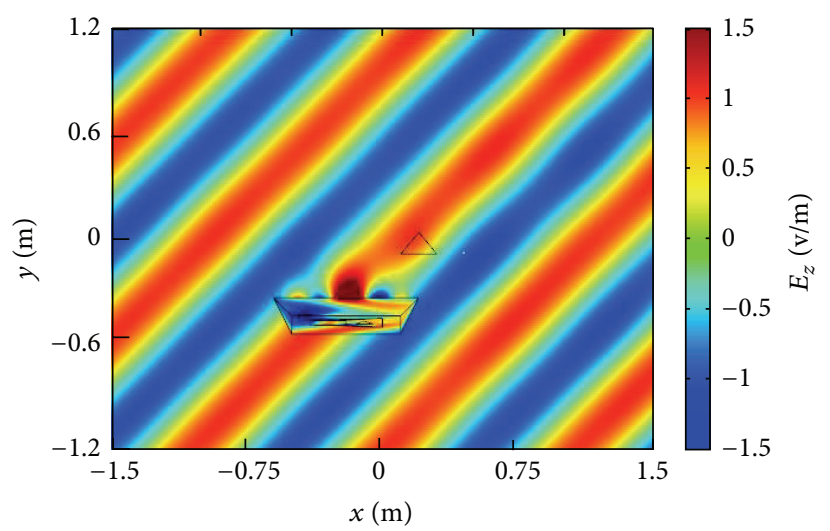

(c)

FIGURE 5: Electric field distributions for the trapezoid complementary illusion cloak: (a) without target object; (b) with target object for a normally incident wave; (c) with target object for an obliquely incident wave.

antiobject in region VI with two coordinate transformations along two different directions, as shown in Figure 3. With two coordinate transformations, the sizes of the antiobject are greatly reduced. Also we can see that the cloak used to hide target object is the complementary cloak generated by the illusion. Hence, unlike the conventional complementary cloak, the relative position between the whole device and the target object can be arbitrary in the proposed complementary illusion cloak.

\section{Simulations and Discussions}

A finite element solver (COMSOL Multiphysics) is used to verify and demonstrate the performance of the proposed complementary illusion cloak. Without loss of generality, $\mathrm{TE}_{z}$ wave (the electric field perpendicular to the $z$ direction) is considered so that the material parameters including $\varepsilon_{z z}^{\prime}, \mu_{x x}^{\prime}$, $\mu_{x y}^{\prime}$, and $\mu_{y y}^{\prime}$ are solved in the simulations.

As the first example, consider a plane wave with the operating frequency of $900 \mathrm{MHz}$ incident on a nonmagnetic triangle object outside a trapezoid complementary cloak, as shown in Figure 4. The background material is assumed to be air and the relative permittivity of a triangle object is 2. Geometry parameters corresponding to the cloak in Figure 2(a) are chosen as $x_{1}=0.4 \mathrm{~m}, x_{2}=0.4 \mathrm{~m}, x_{3}=$ $0.2 \mathrm{~m}, y_{1}=0.1 \mathrm{~m}$, and $y_{2}=0.2 \mathrm{~m}$, respectively, and thus the electric size of the cloak is $2.4 \lambda \times 1.2 \lambda$ ( $\lambda$ is the free-space wavelength). With the complementary cloak, we clearly see from Figure 4 that the triangle object is hidden, and meanwhile it can receive the incident electromagnetic wave so that the communication between the object and the outer space can be achieved. The size of the required antiobject is compressed along $x$ direction due to the use of the coordinate transformation along $x$ direction, while its size along $y$ direction keeps unchanged. Hence the resultant cloak is in a large size. Moreover, it can be seen from Figure 4 that the complementary cloak can be only placed along $x$ axis to hide the target object due to the use of the coordinate transformation along $x$-axis.

In order to simultaneously reduce the dimensions of the antiobject along $x$ and $y$ directions, a complementary illusion cloak is designed. Parameters associated with the cloak in Figure 2(b) are chosen as $x_{4}=0.2 \mathrm{~m}, x_{5}=0.1 \mathrm{~m}, x_{6}=0.5 \mathrm{~m}$, $x_{7}=0.6 \mathrm{~m}, y_{3}=0.3 \mathrm{~m}, y_{4}=0.3 \mathrm{~m}, y_{5}=0.4 \mathrm{~m}$, and $y_{6}=0.5 \mathrm{~m}$, respectively. A plane wave with the frequency of $300 \mathrm{MHz}$ is incident along $x$-axis, and therefore the electric size of the cloak is $0.8 \lambda \times 0.8 \lambda$. With proposed complementary illusion cloak, the trapezoid complementary cloak in first example can be illusively generated. Figure 5(a) demonstrates the electric field distribution without the nonmagnetic target object. Figures 5(b) and 5(c) show the electric field distributions with the nonmagnetic target object for the normally and obliquely incident waves, respectively. Here the relative permittivity of the target object is 2. A good invisibility 


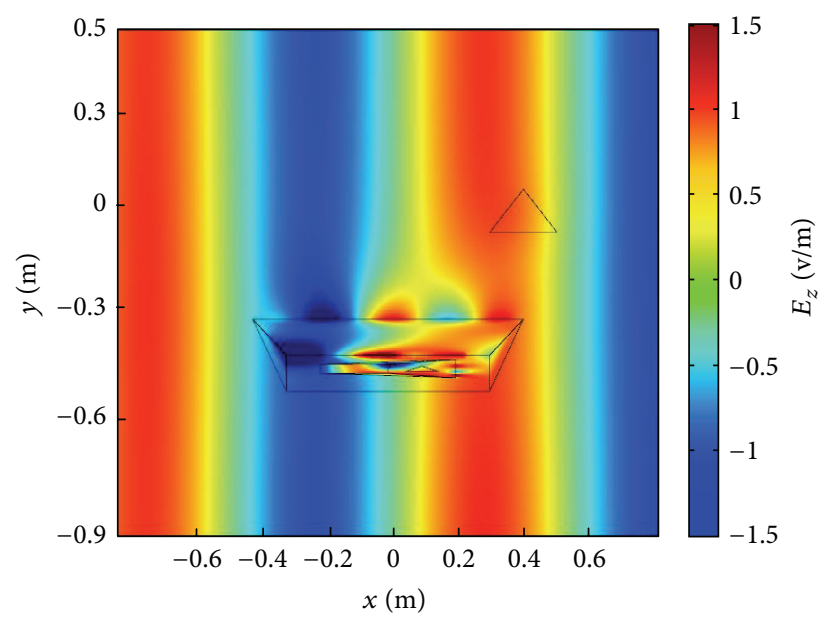

(a)

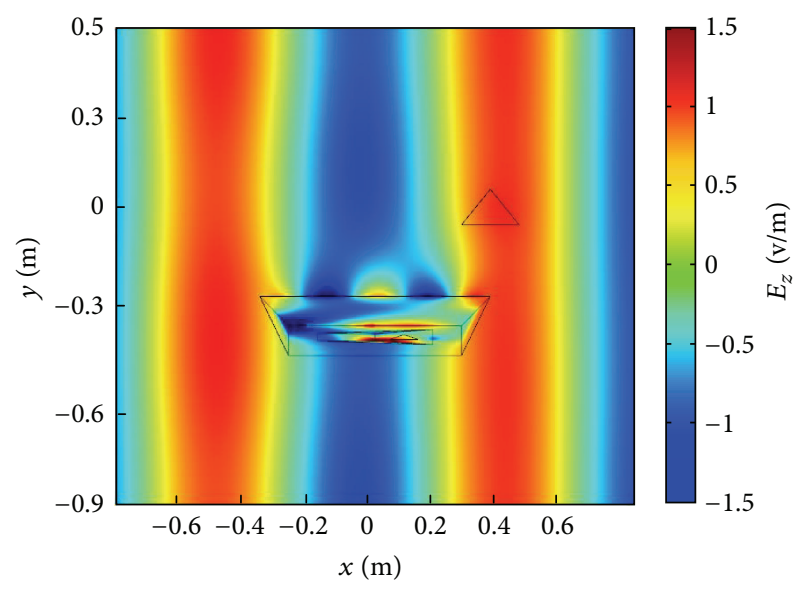

(c)

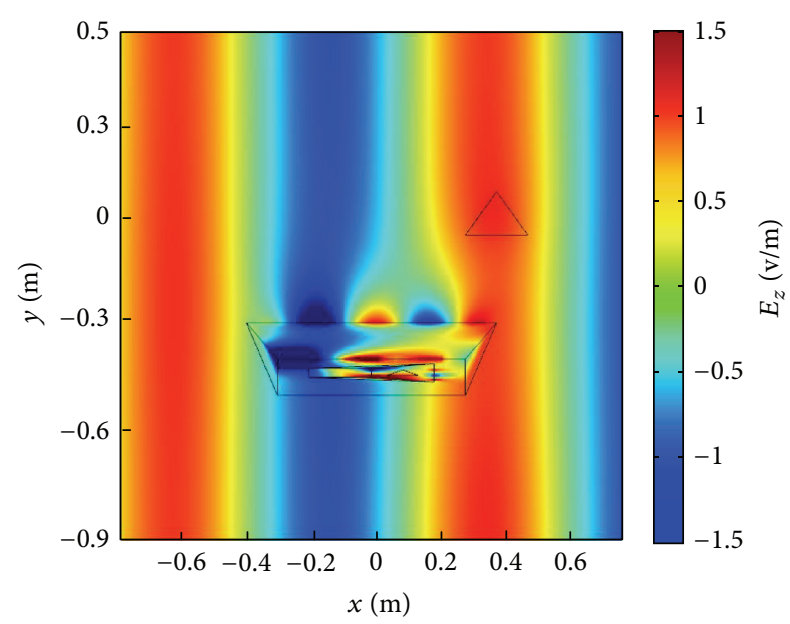

(b)

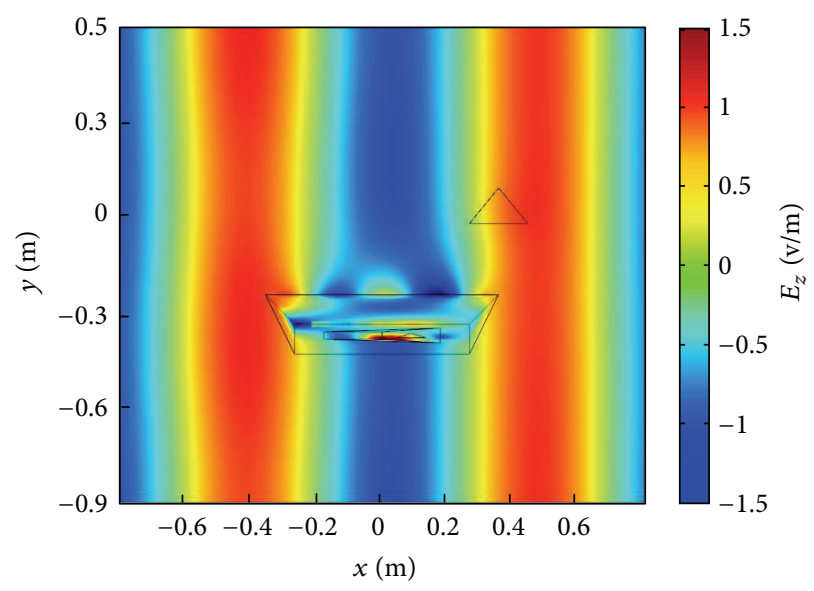

(d)

FIGURE 6: Electric field distributions of the trapezoid complementary illusion cloak for the incident plane waves with different initial phases: (a) $0^{\circ}$; (b) $30^{\circ}$; (c) $60^{\circ}$; (d) $90^{\circ}$.

can be obtained by using the complementary illusion cloak. Moreover, it can clearly be seen from Figures 5(b) and 5(c) that the sizes of the required antiobject are greatly reduced along both $x$ and $y$ directions. Moreover, with the use of two coordinate transformations, the complementary illusion cloak can be placed along $y$-axis compared to the case of the above complementary cloak. In addition, Figure 6 shows the electric field distributions when the plane waves with the initial phases of $0^{\circ}, 30^{\circ}, 60^{\circ}$, and $90^{\circ}$ are incident on the trapezoid complementary illusion cloak. According to Figure 6, good invisibility can be observed.

In the following, we consider a complementary illusion cloak with the following geometric parameters: $x_{1}=1 \mathrm{~cm}$, $x_{2}=0.75 \mathrm{~cm}, x_{3}=0.5 \mathrm{~cm}, x_{4}=0.75 \mathrm{~cm}, x_{5}=0.5 \mathrm{~cm}$, $x_{6}=1 \mathrm{~cm}, x_{7}=1.25 \mathrm{~cm}, y_{1}=0.25 \mathrm{~cm}, y_{2}=0.5 \mathrm{~cm}$, $y_{3}=0.75 \mathrm{~cm}, y_{4}=0.75 \mathrm{~cm}, y_{5}=1 \mathrm{~cm}$, and $y_{6}=1.25 \mathrm{~cm}$. Figure 7 shows invisibility of the cubic nonmagnetic object with relative permittivity of 4 , when the plane waves with the frequencies of $2 \mathrm{GHz}, 2.5 \mathrm{GHz}$, and $3 \mathrm{GHz}$ are incident from the left to the right on the complementary illusion cloak.
Finally, we use the trapezoid complementary illusion cloak to hide a nonmagnetic target object with the boundary of $R(t)=0.05+0.005 \cdot \sin (t)+0.001 \cdot \sin (2 t)$ and the relative permittivity of 2 . Parameters of designed complementary illusion cloak and generated complementary cloak are as follows: $x_{1}=0.4 \mathrm{~m}, x_{2}=0.1 \mathrm{~m}, x_{3}=0.05 \mathrm{~m}, x_{4}=0.1 \mathrm{~m}, x_{5}=$ $0.05 \mathrm{~m}, x_{6}=0.15 \mathrm{~m}, x_{7}=0.2 \mathrm{~m}, y_{1}=0.2 \mathrm{~m}, y_{2}=0.25 \mathrm{~m}$, $y_{3}=0.3 \mathrm{~m}, y_{4}=0.3 \mathrm{~m}, y_{5}=0.4 \mathrm{~m}$, and $y_{6}=0.5 \mathrm{~m}$. TE $z$ wave with the frequency of $400 \mathrm{MHz}$ is incident along $x$-axis. In this example, the electric size of the cloak is $0.4 \lambda \times 1.07 \lambda$. Figure 8 shows the good performance of the proposed cloak including small size, invisibility, and arbitrary position.

\section{Conclusion}

In this paper, a trapezoid complementary illusion invisibility cloak is designed according to the complementary cloak and illusion theory. A trapezoid complementary cloak is first achieved by a coordinate transformation along one direction. With the illusion theory, a new illusion cloak device 


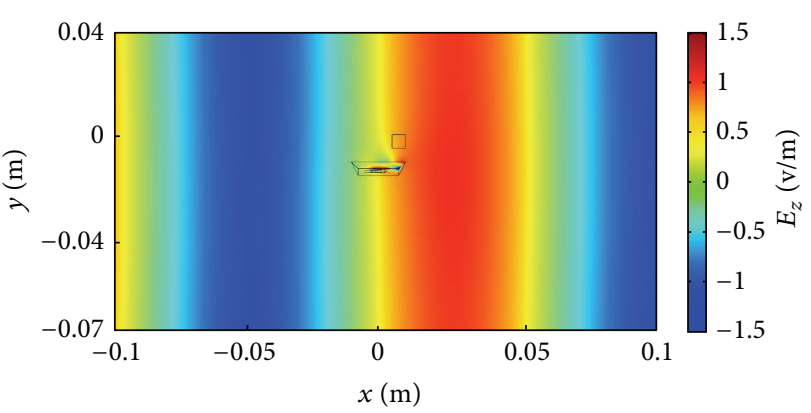

(a)

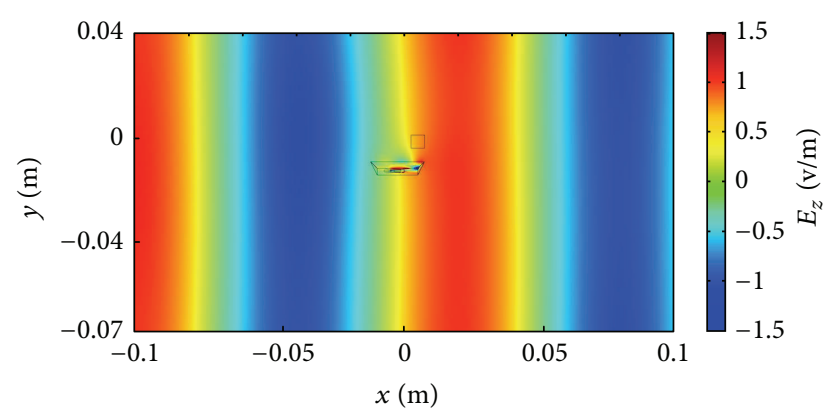

(b)

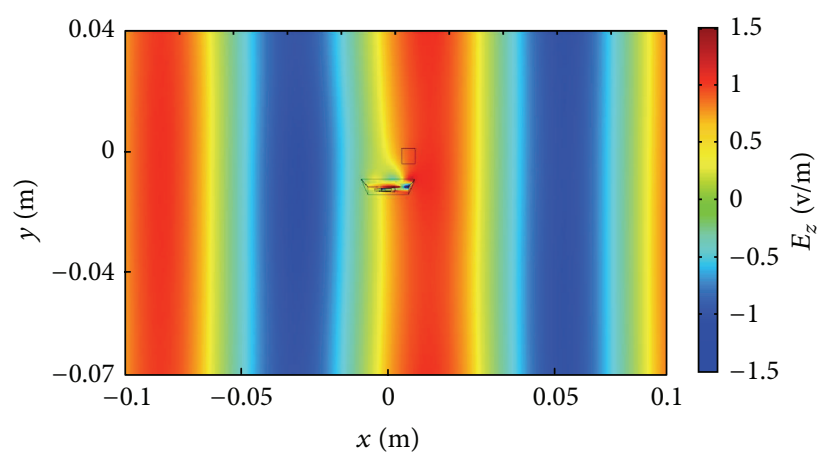

(c)

FIGURE 7: Electric field distribution of the trapezoid complementary illusion cloak for the incident plane wave with different frequencies: (a) $2 \mathrm{GHz}$; (b) $2.5 \mathrm{GHz}$; (c) $3 \mathrm{GHz}$.

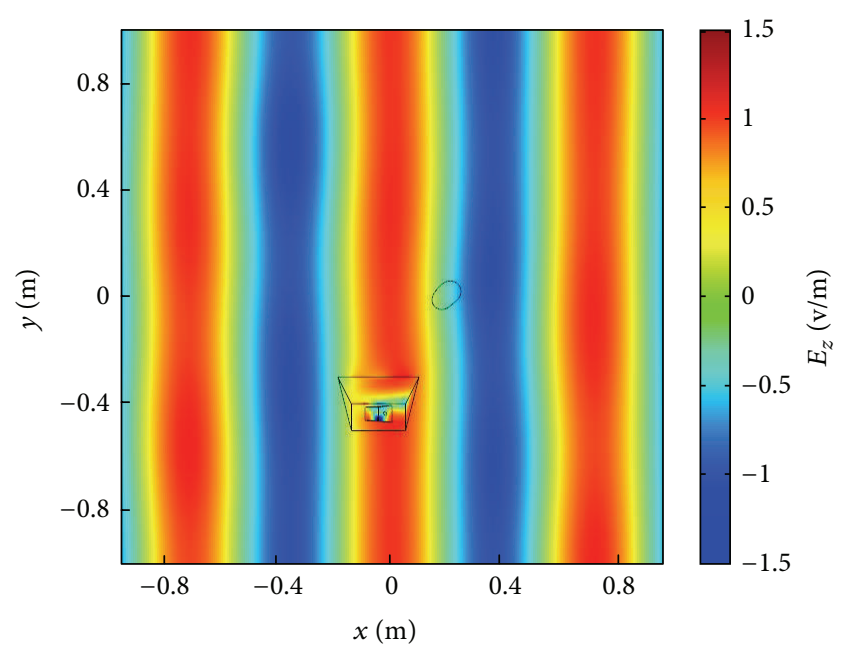

FIGURE 8: Electric field distribution of an object with an arbitrary boundary.

based on a coordinate transformation along another direction is further generated to cloak the target object. With the coordinate transformations along two different directions, the location of the complementary illusion cloak device can be arbitrarily chosen, and the sizes of the required antiobject are greatly reduced to cloak a target object with arbitrary size and shape. The good agreement between the simulation and the theory shows good performance of the proposed complementary illusion cloak.

\section{Conflict of Interests}

The authors declare that there is no conflict of interests regarding the publication of this paper.

\section{Acknowledgments}

This work is supported partly by the Program for the New Scientific and Technological Star of Shaanxi Province (no. 2013KJXX-66), Natural Science Basic Research Plan in Shaanxi Province (no. 2013JZ019 and no. 2015JM6292), Technology Innovation Research Project of the CETC, and Fundamental Research Funds for the Central Universities (no. SPSZ031410).

\section{References}

[1] J. B. Pendry, D. Schurig, and D. R. Smith, "Controlling electromagnetic fields," Science, vol. 312, no. 5781, pp. 1780-1782, 2006.

[2] U. Leonhardt, "Optical conformal mapping," Science, vol. 312, no. 5781, pp. 1777-1780, 2006.

[3] D. Schurig, J. B. Pendry, and D. R. Smith, "Calculation of material properties and ray tracing in transformation media," Optics Express, vol. 14, no. 21, pp. 9794-9804, 2006. 
[4] S. A. Cummer, B.-I. Popa, D. Schurig, D. R. Smith, and J. B. Pendry, "Full wave simulations of electromagnetic cloaking structures," Physical Review E, vol. 14, pp. 9794-9804, 2006.

[5] W. X. Jiang, T. J. Cui, X. M. Yang, Q. Cheng, R. Liu, and D. R. Smith, "Invisibility cloak without singularity," Applied Physics Letters, vol. 93, no. 19, Article ID 194102, 2008.

[6] M. Rahm, S. A. Cummer, D. Schurig, J. B. Pendry, and D. R. Smith, "Optical design of reflectionless complex media by finite embedded coordinate transformations," Physical Review Letters, vol. 100, no. 6, Article ID 063903, 2008.

[7] G. W. Milton, M. Briane, and J. R. Willis, "On cloaking for elasticity and physical equations with a transformation invariant form," New Journal of Physics, vol. 8, article 248, 2006.

[8] W. X. Jiang, J. Y. Chin, Z. Li, Q. Cheng, R. Liu, and T. J. Cui, "Analytical design of conformally invisible cloaks for arbitrarily shaped objects," Physical Review E: Statistical, Nonlinear, and Soft Matter Physics, vol. 77, no. 6, Article ID 066607, 2008.

[9] T. Ergin, N. Stenger, P. Brenner, J. B. Pendry, and M. Wegener, "Three-dimensional invisibility cloak at optical wavelengths," Science, vol. 328, no. 5976, pp. 337-339, 2010.

[10] W. Cai, U. K. Chettiar, A. V. Kildishev, and V. M. Shalaev, "Optical cloaking with metamaterials," Nature Photonics, vol. 1, no. 4, pp. 224-227, 2007.

[11] T. Yang, H. Y. Chen, X. D. Luo, and H. R. Ma, "Superscatterer: enhancement of scattering with complementary media," Optics Express, vol. 16, no. 22, pp. 18545-18550, 2008.

[12] M. Rahm, D. Schurig, D. A. Roberts, S. A. Cummer, D. R. Smith, and J. B. Pendry, "Design of electromagnetic cloaks and concentrators using form-invariant coordinate transformations of Maxwell's equations," Photonics and NanostructuresFundamentals and Applications, vol. 6, no. 1, pp. 87-95, 2008.

[13] I. Gallina, G. Castaldi, V. Galdi, A. Alù, and N. Engheta, "General class of metamaterial transformation slabs," Physical Review B-Condensed Matter and Materials Physics, vol. 81, no. 12, Article ID 125124, 2010.

[14] D. Schurig, J. J. Mock, B. J. Justice et al., "Metamaterial electromagnetic cloak at microwave frequencies," Science, vol. 314, no. 5801, pp. 977-980, 2006.

[15] A. Alù and N. Engheta, "Multifrequency optical invisibility cloak with layered plasmonic shells," Physical Review Letters, vol. 100, no. 11, Article ID 113901, 2008.

[16] H. Y. Chen and C. T. Chan, "Transformation media that rotate electromagnetic fields," Applied Physics Letters, vol. 90, no. 24, Article ID 241105, 2007.

[17] Y. Lai, H. Chen, Z.-Q. Zhang, and C. T. Chan, "Complementary media invisibility cloak that cloaks objects at a distance outside the cloaking shell," Physical Review Letters, vol. 102, no. 9, Article ID 093901, 2009.

[18] J. B. Pendry, "Negative refraction makes a perfect lens," Physical Review Letters, vol. 85, no. 18, article 3966, 2000.

[19] C. F. Yang, J. J. Yang, M. Huang, Z. Xiao, and J. H. Peng, "An external cloak with arbitrary cross section based on complementary medium and coordinate transformation," Optics Express, vol. 19, no. 2, pp. 1147-1157, 2011.

[20] Y.-D. Xu, L. Gao, and H.-Y. Chen, "Cloak an illusion," Frontiers of Physics in China, vol. 6, no. 1, pp. 61-64, 2011.

[21] Y. Lai, J. Ng, H. Chen et al., "Illusion optics: the optical transformation of an object into another object," Physical Review Letters, vol. 102, no. 25, Article ID 253902, 2009.
[22] F.-F. Huo, L. Li, T. Li, Y.-M. Zhang, and C.-H. Liang, "External invisibility cloak for multiobjects with arbitrary geometries," IEEE Antennas and Wireless Propagation Letters, vol. 13, pp. 273276, 2014. 

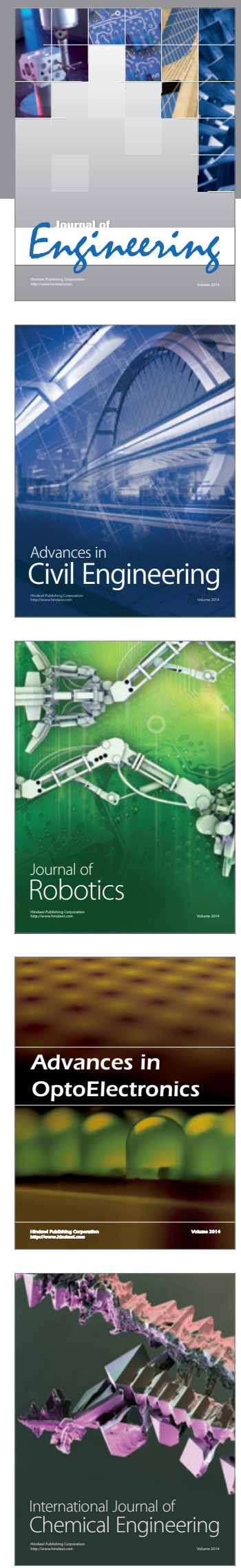

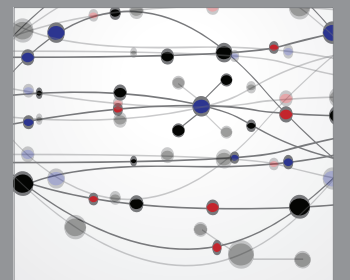

The Scientific World Journal
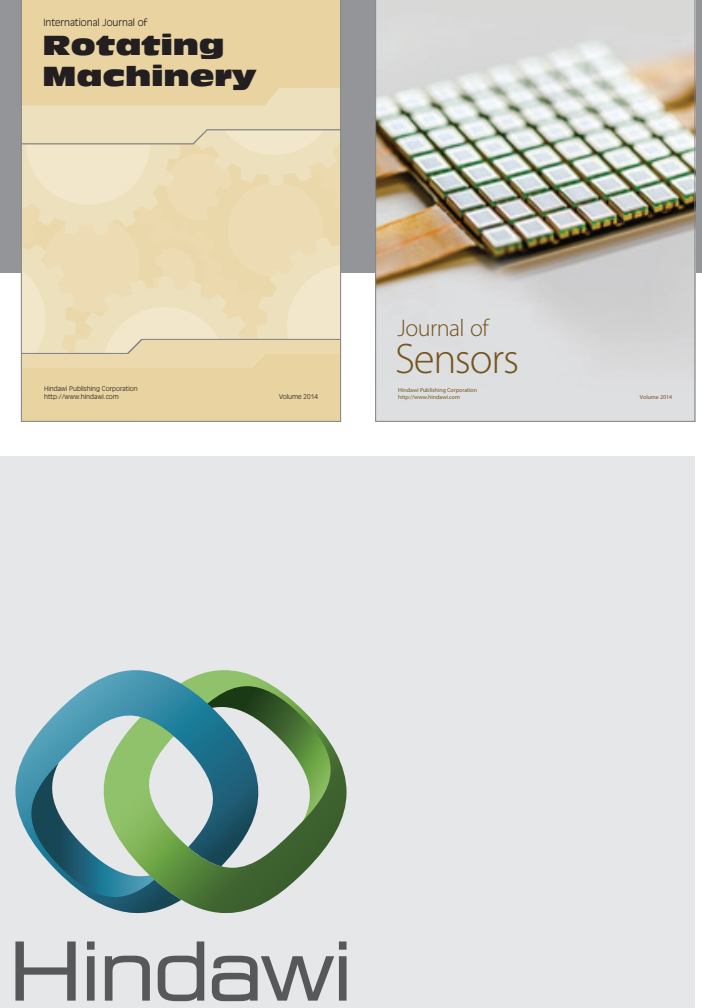

Submit your manuscripts at http://www.hindawi.com
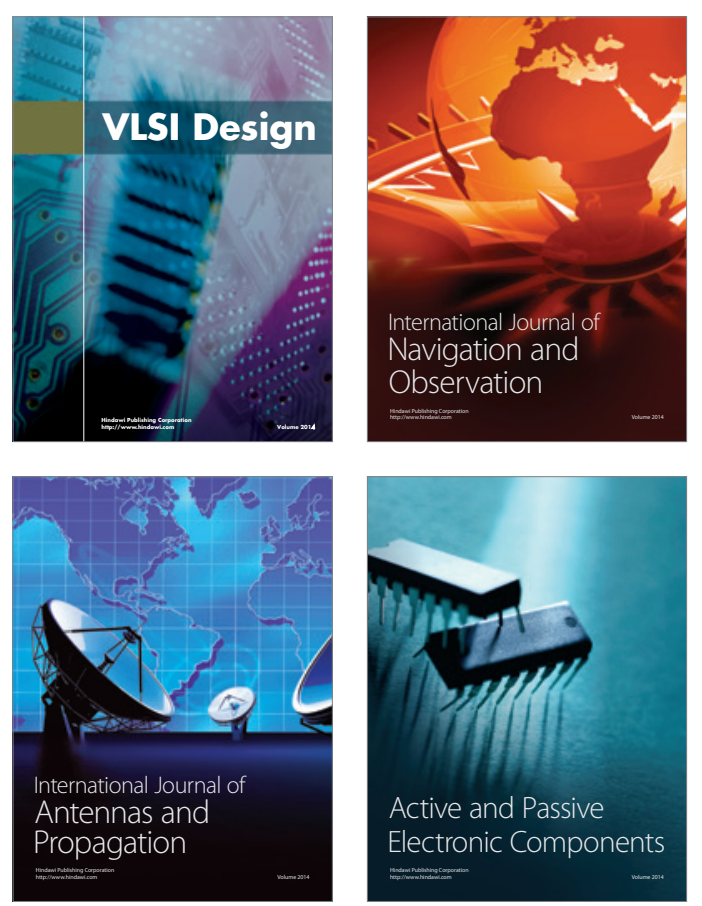
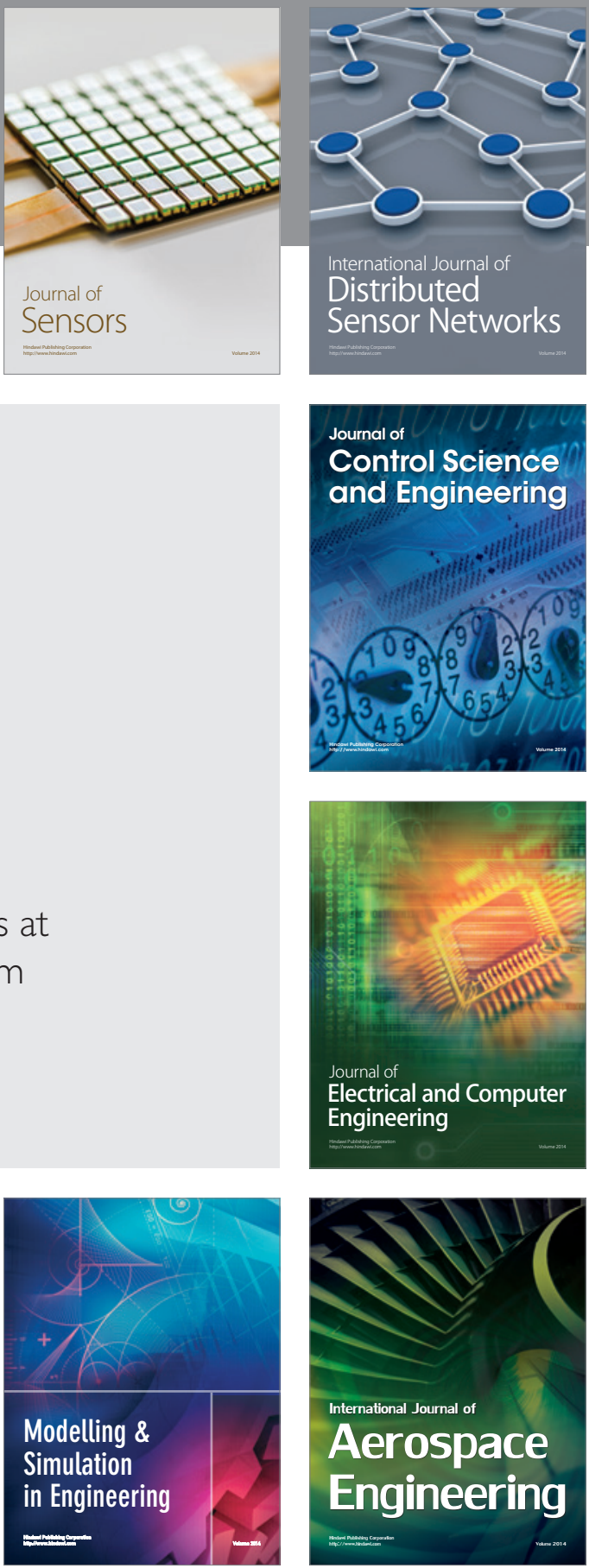

Journal of

Control Science

and Engineering
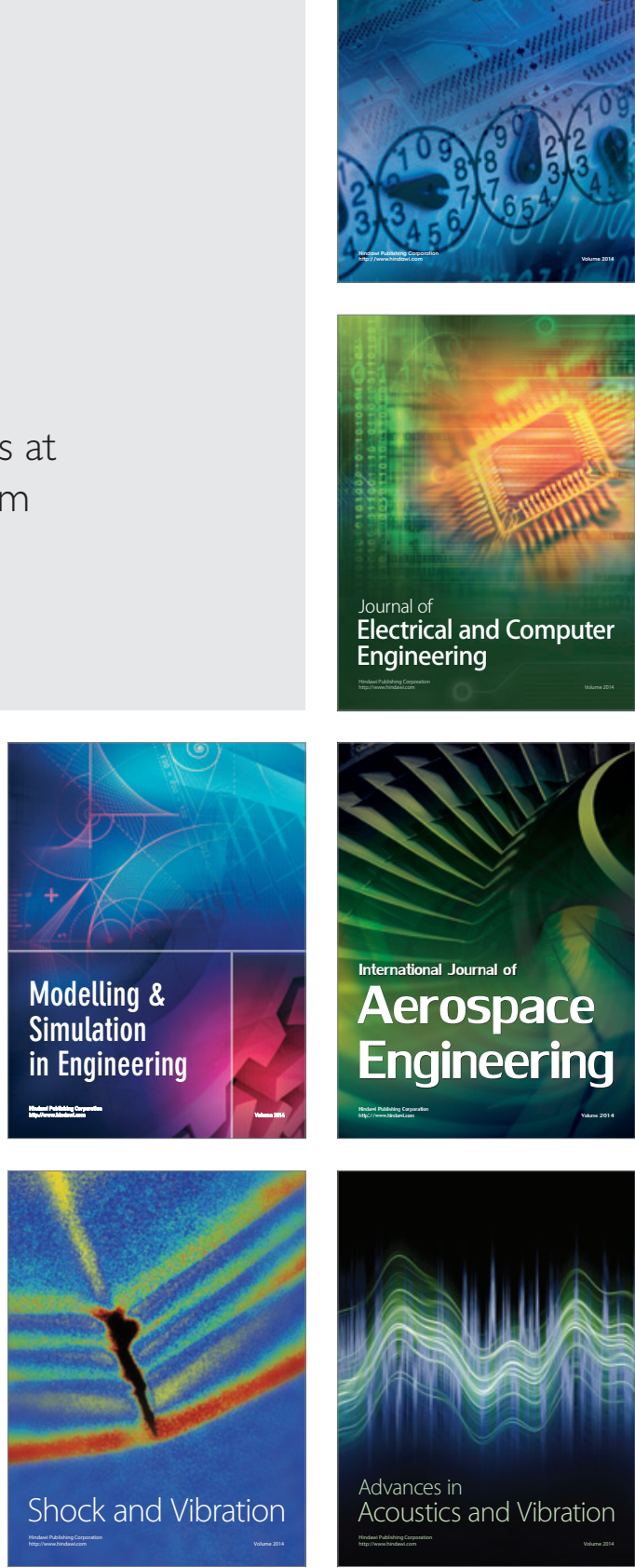